\title{
REVIEW ARTICLE
}

\section{Combination of anti-angiogenic therapy Apatinib and immune therapy potentiate tumor microenvironment} Chunyi Gao, Tianhui Hu*

Cancer Research Center, School of Medicine, Xiamen University, Xiamen 361102, Fujian Province, China. E-mail: thu@xmu.edu.cn

\begin{abstract}
Tumor immune therapy, especially anti-programmed cell death ligand-1/programmed cell death-1 (PD-L1/PD-1) treatment, is currently the focus of substantial attention. However, despite its enormous successes, the overall response rate of cancer immunotherapy remains suboptimal. There is an increased interest in combining PD-L1/PD-1 treatment with anti-angiogenic drug Apatinib to enhance antitumor effect. Presently available data seem to suggest that Apatinib may exert immune suppressive effects to make the PD-L1/PD-1 treatment works. Here, we review the extensive tumor microenvironment immune modulatory effects from antiangiogenic agents Apatinib in order to supporting VEGFR2 targettherapies in clinical trials are existing.
\end{abstract}

Keywords: Apatinib; Tumor Environment; PD-1/PD-L1; Tumor Immune Therapy

\section{ARTICLE INFO}

Received 13 September 2021

Accepted 9 November 2021

Available online 16 November 2021

\section{COPYRIGHT}

Copyright @ 2021 Chunyi Gao, et al. EnPress Publisher LLC. This work is licensed under the Creative Commons Attribution-NonCommercial 4.0 International License (CC BY-NC 4.0).

https://creativecommons.org/licenses/by-nc/ $4.0 /$

\section{Introduction of Apatinib}

Apatinib is a small-molecule antiangiogenic targeted drug developed by Jiangsu Hengrui Pharmaceutical Co., Ltd. It was listed in the treatment of gastric cancer in 2014. Furthermore, the drug also shows great potential in the treatment of multiple other solid tumors ${ }^{[1,2]}$. The main mechanism of action of Apatinib is the competitive binding of the vascular endothelial growth factor receptor2 (VEGFR-2) intracellular tyrosine ATP binding site. Then selectively inhibiting tyrosine kinase activity and blocking signaling after vascular endothelial growth factor (VEGF) binding, resulting in potent inhibition of tumor angiogenesis. VEGFR-2 is a high-affinity receptor for VEGF and, together with VEGFR-1 and VEGFR-3, forms the Flt subfamily of receptor tyrosine kinases (RTK). VEGFR-1 binds to VEGF with a higher affinity than VEGFR-2, but VEGFR-1 exerts weak tyrosine phosphorylation of VEGF. Thus, VEGFR-2 is the primary RTK that can mediate VEGF signaling in endothelial cells and drive VEGF-mediated angiogenic ${ }^{[3]}$. Moreover, VEGFR-2 is the major regulator of endothelial cell proliferation, development, migration, angiogenesis, and budding ${ }^{[4]}$. Therefore, Apatinib may have better therapeutic potential than other antiangiogenic agents such as ramoximab, bevacizumab and sunitinib ${ }^{[5]}$. In addition, Apatinib can sensitize drug-resistant tumor cells to chemotherapeutic drugs and reverse multidrug resistance (MDR) caused by ATP binding cassette transporter (ABC) protein ${ }^{[6]}$. It can also stimulate tumor cell apoptosis, dinhibit cell proliferation and enhance the efficacy of conventional chemotherapy drugs to play an anti-cancer role. 
In recent years, the clinical application and related mechanism of Apatinib in other tumors have also been widely concerned and explored ${ }^{[7-9]}$. However, so far, the clinical application has generally focused on inhibiting abnormal angiogenesis. This review summarizes the unique mechanism of action of Apatinib, based on preclinical and clinical findings, the molecular mechanism behind the pathway of interaction with Apatinib was reexplored. Specifically, Apatinib includes both wellknown anti-angiogenic effects and novel immunomodulatory mechanisms. It can promote the clinical understanding of molecular biology and immunology, and help to expand the scope and prospect of Apatinib treatment.

\section{Immunotherapy and VEGF/VEGFR targets}

Immune checkpoint inhibitors (ICIS) can break through the bottleneck of tumor immunosuppression, restore the suppressed immune activity in tumor patients, and promote immune cells to recognize and then kill tumor cells. It provide a new idea for immunotherapy ${ }^{[10,11]}$. In recent years, programmed cell death 1 (PD-1) antibody, programmed cell death ligand 1 (PD-L1) antibody, and cytotoxic $\mathrm{T}$ lymphocyte-associated protein 4
(CTLA-4) antibody and other representative immunotherapies have become an important means of tumor treatment ${ }^{[12]}$. Especially for antibodies against PD-1/PD-L1 have achieved significant clinical efficacy in the therapy of malignant tumors such as lung cancer, kidney cancer, and melanoma. The high expression of PD-L1 may be a molecular marker of poor prognosis of cancer, and it is also the basis for clinical treatment with anti- PD-1/PDL1.

However, the anti-PD-1/PD-L1 treatment also has its limitations, the main problem is the low proportion of beneficiaries, the response rate of single drug use is generally between $10 \%$ and $40 \%$, and not all tumor types are effective ${ }^{[13]}$. The main reason for the low benefit ratio is that the efficacy of immunotherapy highly depends on the Tumor Microenvironment (TME). The TME is mainly composed of the vasculature, the extracellular matrix, other non-malignant cells around the tumor, as well as the complex signaling molecular networks that maintain internal connections in the microenvironment. These components not only could promote the growth and reproduction of the tumor cells but also induce them to invade and metasta$\operatorname{size}^{[14]}$.
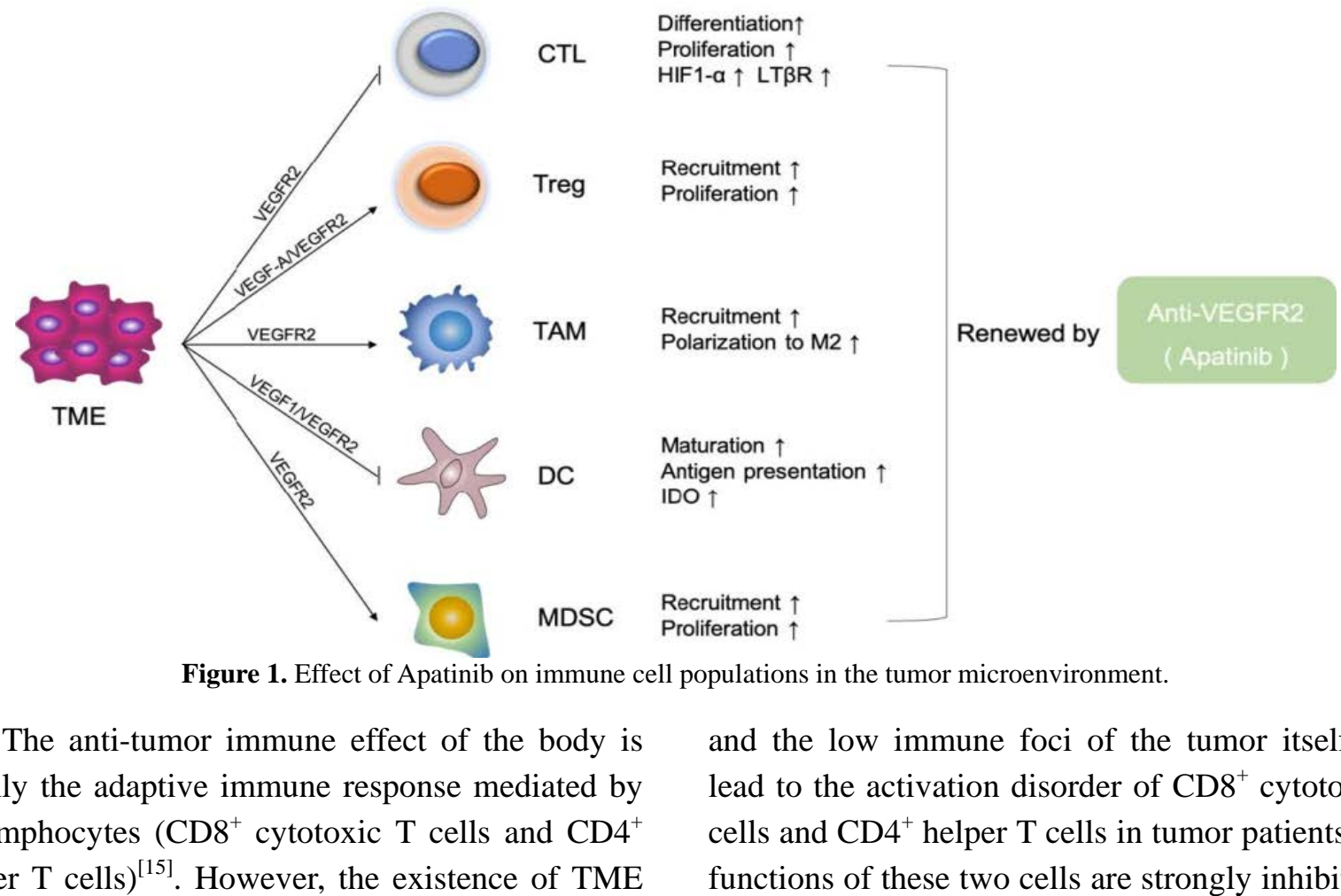

Proliferation $\uparrow$

The anti-tumor immune effect of the body is mainly the adaptive immune response mediated by $\mathrm{T}$ lymphocytes $\left(\mathrm{CD}^{+}\right.$cytotoxic $\mathrm{T}$ cells and $\mathrm{CD} 4^{+}$ helper $\mathrm{T}$ cells $)^{[15]}$. However, the existence of TME and the low immune foci of the tumor itself will lead to the activation disorder of $\mathrm{CD}^{+}$cytotoxic $\mathrm{T}$ cells and $\mathrm{CD} 4^{+}$helper $\mathrm{T}$ cells in tumor patients. The functions of these two cells are strongly inhibited in 
the tumor microenvironment, which is one of the main obstacles to anti-PD-1/PD-L1 immunothera$\mathrm{py}^{[16]}$. And it is also one of the main reasons for the low response rate of immunotherapy. Therefore, inhibiting of the negatively regulated recruitment of immune cells in the tumor microenvironment, or inhibiting the secretion of anti-inflammatory factors. That may make the effect of the tumor immunotherapy more significant. In recent years, more studies and clinical trials have found that the combined use of anti-tumor angiogenesis drugs and PD-1/PD-L1 antibodies could effectively improve the efficacy of immunotherapy. Its mechanism is closely related to the remodeling of the tumor microenvironment ${ }^{[17,18]}$. Next, we will describe the effects of the antiangiogenic agent Apatinib on the antitumor function of immune cells in preclinical and clinical trials in detail (Figure 1).

\section{Effect of Apatinib on the immune microenvironment}

\subsection{Effect of Apatinib on effector $T$ cells}

In the terms of $\mathrm{T}$ cells, these unusual tumor vascular networks provide an attack barrier for tumor cells. The abnormal vascular network is mostly immature neovascularization, which does not have the tissue structure of normal blood vessels, so it cannot perform a normal function. Neovascular endothelial cells are loosely connected and lack the support of pericytes and basal cells. Therefore, tissue fluid seeps from the abnormal vascular network into the tumor microenvironment to further compress the blood vessels, and the blood cannot transport oxygen and nutrients to the interior of the tumor, resulting in hypoxia and necrosis in the interior of the tumor. Similarly, most killer T cells cannot infiltrate into the tumor, so their killing effect is inhibited. A small number of $\mathrm{T}$ cells crossing vascular endothelial cells into the tumor microenvironment still cannot kill tumor cells. The reason is the increased expression of immunosuppressive marker PD-L1 on the surface of tumor cells under long-term hypoxia. Therefore, only after anti-vascular treatment, killer T cells infiltrate into the tumor area, and then anti-PD-1 treatment can exert a good curative effect ${ }^{[19]}$.
VEGF increased the infiltration of regulatory $\mathrm{T}$ cells in the microenvironment and promoted the failure of $\mathrm{CD}^{+} \mathrm{T}$ cells. TOX is a transcription factor of $\mathrm{T}$ cell development and plays an important role in $\mathrm{T}$ cell maturation. In the tumor microenvironment, VEGF up-regulates the expression of TOX in $\mathrm{CD}^{+} \mathrm{T}$ cells and activates the failure-related signal pathway of $\mathrm{T}$ cells. Anti VEGF treatment can reverse this phenomenon. VEGFR-2 ramucirumab promotes the increase of the proportion of $\mathrm{CD}^{+} \mathrm{T}$ cells in peripheral blood of patients with gastric cancer ${ }^{[20]}$. More studies have found that VEGFR-2 blocked HIF1- $\alpha$ of $\mathrm{CD}^{+} \mathrm{T}$ cells activation of the pathway leads to increased secretion of anti-inflammatory factors ${ }^{[21]}$. In the mouse lung cancer model, the use of low-dose Apatinib increased the infiltration of $\mathrm{CD}^{+}{ }^{\mathrm{T}}$ cells in tumors ${ }^{[22]}$. Comprehensive studies have shown that blocking VEGFR-2 could increase the proportion and activity of $\mathrm{CD8}^{+} \mathrm{T}$ cells ${ }^{[23,24]}$. Moreover, low-dose VEGFR-2 target drug (DC101) is more conducive to promoting the tumor infiltration of $\mathrm{CD}^{+}$and $\mathrm{CD}^{+} \mathrm{T}$ cells ${ }^{[25]}$. In spontaneous breast cancer mice, DC101 inhibited tumor growth by increasing the proportion of $\mathrm{CD}^{+} \mathrm{T}$ cells ${ }^{[25,26]}$. In the exploration of its mechanism, ELIZABETH ALLEN et al. proposed that combination therapy of anti-VEGFR-2 and anti-PD-L1 antibodies in mice models of breast, pancreatic and glioblastoma can induce the growth, and proliferation of endothelial micro veins in the tumor microenvironment ${ }^{[19]}$. Endothelial micro veins promote lymphocyte infiltration and activity through the activation of the lymphotoxin $\beta$ receptor (LT $\beta \mathrm{R}$ ) on the lymphocyte surface. Using LT $\beta \mathrm{R}$ directly agonist can enhance the activity of cytotoxic T cells (CTL), so that PD-L1 antibody can further improve its antitumor efficacy after combined treatment with anti-VEGFR-2.

\subsection{Effect of Apatinib on regulatory T cells}

Regulatory $\mathrm{T}$ cells, namely $\mathrm{CD} 4{ }^{+} \mathrm{CD} 25^{+}$Treg, account for $5 \% \sim 10 \%$ of the total $\mathrm{T}$ cells and are key negative regulatory cells in the tumor immune microenvironment. Classic sub-Types are divided into natural regulatory $\mathrm{T}$ cells (nTregs) differentiated from the thymus and inducible regulatory $\mathrm{T}$ cells (iTregs) induced by antigens or other cytokines. 
Foxp3 is the functional activity index of Treg ${ }^{[27]}$. Suzuki $\mathrm{H}$ et al. found that $\mathrm{Foxp}^{+} \mathrm{CD}^{+} \mathrm{T}$ cells specifically express VEGFR-2, and VEGFR-2 ${ }^{+}$ $\mathrm{CD}^{+} \mathrm{T}$ cells inhibit the proliferation of VEGFR-2 ${ }^{-}$ $\mathrm{CD} 4^{+} \mathrm{T}$ cells ${ }^{[28]}$. TGF- $\beta$ further increases the number of VEGFR2 ${ }^{+}$Tregs by promoting VEGF-A secretion $^{[28]}$. Targeting VEGFR can effectively reduce the proportion of Treg, weaken its immunosuppressive activity and enhance the antitumor immune response of effector T cells. VEGF-A directly promotes the proliferation of Treg, and blocking VEGF-A/VEGFR-2 pathway can reverse this phenomenon $^{[29]}$.

TADA Y et al. found that PD-L1 + $\mathrm{CD}^{+} \mathrm{T}$ cell infiltration in peripheral blood increased after targeted VEGFR-2 treatment in patients with gastric cancer $^{[20]}$. The proportion of Treg decreased. Progression-free survival was longer in patients with low expression of VEGFR-2 ${ }^{+}$Treg than in patients with high expression. According to more studies, in the study of mouse colon cancer, PD-1 antibody increased the proportion of Foxp3 $3^{+}$Treg in tumor tissue $^{[30]}$. This phenomenon can recover by combined use of VEFR-2 antibody, and the combined use increased IFN- $\gamma$ in tumor homogenate And TNF- $\alpha$ Level. In hepatoma mice, VEGF-2 antibody (DC101) increased the CTL/Treg ratio in tumor tissues $^{[31]}$.

\subsection{Effect of Apatinib on macrophages}

Tumor-associated macrophages (TAM) are the main inflammatory cell population in solid tumors and have an important impact on the composition of TME. Plasticity is an important concept in TAM research, that is, TAM can differentiate into M1/M2 types according to different stimuli of TME. M1 macrophages derived from interferon $\gamma($ IFN- $\gamma$ ) and inflammatory cytokines stimulated and secreted by lipopolysaccharide (LPS). Matrix remodeling cytokines activated and released by IL-4 and IL-13 stimulated M2 macrophages. "Remodeling” TAM, that is, transforming M2 into anti-tumor M1, may become a potentially effective strategy for cancer treatment ${ }^{[32]}$.

In the treatment of lung cancer mice, oral low doses of Apatinib increased the proportion of infiltration of total macrophages and reduced the pro- portion of cancer type $\mathrm{CD}_{163}{ }^{+} \mathrm{M} 2$ macrophages $^{[22]}$. In liver cancer mice, small-dose VEGFR-2 antibody (DC101) treatment significantly increased the number of $\mathrm{F} 4 / 80^{+} \mathrm{CD} 80^{+}$and $\mathrm{CD} 6^{+} \mathrm{M} 1$ macrophages infiltrated in the tumor and reduced the number of $\mathrm{F} 4 / 80^{+} \mathrm{CD} 206^{+} \mathrm{M} 2$ macrophages after combined anti-PD-1 treatment.

\subsection{Effect of Apatinib on DCs}

Antigen presentation is a prerequisite for antitumor immune response. Immature dendritic cells (DCs) lack effective antigen presentation function, resulting in immune tolerance ${ }^{[33]}$. Several studies have reported that VEGF inhibits the maturation of DCs in vitro and vivo by activating NF- $\mathrm{KB}$. ALFARO C et al. believed that renal cancer cells inhibit the maturation and differentiation of DCs under hypoxia, which can reverse by Sunitinib, a tyrosine kinase inhibitor of VEGFR- $2^{[34]}$. There are different opinions on the functional blocking sites of VEGF/VEGFR on DCs. Some studies have said that VEGFR-1 is the main receptor for DCs maturation, and the signal transduction of VEGFR-2 is very important for early DCs differentiation in blood, but only has a slight impact on the final DCs maturation ${ }^{[35]}$. Another study shows that VEGF inhibits the ability of mature DCs to present antigens to $\mathrm{T}$ cells, and the DCs dysfunction induced by VEGF is mainly mediated by VEGFR-2. Blocking mAb by anti-VEGFR-2 can reverse DCs dysfunction $^{[36]}$. During the exploration of its mechanism, MARTI LC et al. judged that VEGF up-regulated IDO expression in DCs through VEGF/VEGFR pathway, and IDO further inhibited lymphocyte proliferation $^{[37]}$.

\subsection{Effects of Apatinib on bone mar- row-derived inhibitory cells}

Myeloid-derived suppressor cells (MDSCs) are generally considered as $\mathrm{CD}_{4} 5^{+} \mathrm{CD} 11 \mathrm{~b}^{+} \mathrm{CD}^{+} 3^{+}$ CD14- immunosuppressive cell group. MDSCs were found in tumor, spleen, blood, bone marrow, and liver ${ }^{[38]}$. In TME, MDSCs attenuate the antitumor function of effector $\mathrm{T}$ cells and act on NK cells to reduce their cytokines, and inhibit their cytotoxicity $^{[39]}$. There is a dual expression of VEGFR-1 and VEGFR-2 on the surface of MDSCs. 
VEGFR-2 mediated signal pathway promotes the proliferation of MDSCs ${ }^{[40]}$. VEGF/VEGFR-2 signal transduction also directly promotes the differentiation and migration of MDSCs, recruits MDSCs to tumor sites and inhibits T cell function ${ }^{[26]}$.

\section{Clinical application of Apatin- ib-linked therapy with PD-1/PD-L1}

\subsection{Non-small-cell lung cancer (NSCLC)}

Apatinib showed a significant survival benefit in phase II/IB clinical trials in non-small cell lung cancer, and the effect was better when combined with anti-PD-1. A phase II clinical trial showed that NSCLC patients were treated with 500-750 mg of Apatinib. Among the 38 NSCLC patients evaluated, the objective response rate (ORR) was $13.2 \%$, and the disease control rate (DCR) was 63.2\%. The progression-free survival (PFS) was 3.06 months. Overall survival (OS) was 7.69 months. It shows that Apatinib has a certain role in patients with advanced NSCLC and only has slight adverse reactions $^{[41]}$. The results of another phase IB clinical trial of NSCLC showed that among the 8 NSCLC patients available for analysis, the ORR and DCR of Apatinib were $55.6 \%$ and $88.9 \%$, which were superior to anti-PD-1 monotherapy in the same clinical environment ${ }^{[22]}$.

\subsection{Small-cell lung cancer (SCLC)}

Apatinib also showed therapeutic benefits in SCLC patients. Camrelizumab $200 \mathrm{mg}$ combined with Apatinib $375 \mathrm{mg}$. Of the 47 SCLC patients available for evaluation, ORR reached $34.0 \%$, median PFS was 3.6 months and OS was 8.4 months. Results-oriented patients were classified into platinum-class chemotherapy-sensitive and insensitive groups in SCLC patients. The ORR of platinum chemotherapy-sensitive SCLC patients was $37.50 \%$, the median PFS was 3.6 months, and the OS was 9.6 months. There was no significant difference between SCLC patients and the platinum chemotherapy insensitive group (32.3\%, 2.7 months, 8 months). This study showed that camrelizumab combined with Apatinib showed potential antitumor activity in chemotherapy-sensitive and chemotherapy-resistant SCLC patients.

\subsection{Hepatocellular carcinoma (HCC)}

Jianming $\mathrm{Xu}$ et al. evaluated the efficacy of Apatinib combined with SHR-1210 (anti-PD-1 antibody) in a study on patients with advanced HCC and GC/EGJC at the same time ${ }^{[7]}$. After the end of the trial, 8 of the 16 HCC patients who can be used for evaluation obtained partial remission, and the partial response (PR) was 50.0\%. Including GC/EGJC patients, the ORR of 39 assessable patients was $30.8 \%$. This study shows that the maximum tolerated dose of Apatinib as combination therapy is $250 \mathrm{mg}$, and patients with advanced HCC can obtain practical clinical benefits in combination therapy.

Another study classified patients with HCC. Seventy of these patients did not receive first phase treatment, 120 were ineffective in first phase treatment, and all patients had combined Camrelizumab (anti-PD-1 antibody $200 \mathrm{mg}$ ) and Apatinib 250 $\mathrm{mg} \cdot \mathrm{d}^{-1}$. For the HCC group not receiving phase I treatment, the ORR was $34.3 \%$ and a median PFS of 5.7 months, annual survival was $74.7 \%$. Trials with HCC patients who failed phase I treatment showed that the 120 HCC patients available for evaluation had an ORR of $22.5 \%$ and a median PFS of 5.5 months, annual survival of $68.2 \%$. This study demonstrated that Camrelizumab combined with Apatinib has a survival benefit for patients with advanced liver cancer ${ }^{[42]}$.

\subsection{Gastric or esophagogastric junction can- cer (GC/EGJC)}

In another phase III clinical trial of GC/EGJC patients, the researchers recruited 267 patients ${ }^{[43]}$. Compared with the placebo group, the median OS was significantly prolonged in the Apatinib group (6.5 months vs. 4.7 months, $\mathrm{P}<0.05$ ), and the median PFS was also prolonged (2.6 months vs. 1.8 months, $\mathrm{P}<0.001$ ).

\subsection{Triple-negative breast cancer (TNBC)}

Apatinib in the phase II study of TNBC patients, 30 evaluable patients were given Apatinib $250 \mathrm{mg}$ and SHR-1210 $200 \mathrm{mg}$ continuously. The results showed that the ORR of patients was $43.3 \%$, DCR was $63.3 \%$, and PFS was 3.7 months. The median PFS of patients with partial remission 
was 7.69 months, which was significantly higher than that of patients with TNBC that could not be evaluated clinically (2 months). SHR-1210 combined with Apatinib showed a good therapeutic effect in patients with advanced TNBC ${ }^{[44]}$.

\section{Conclusion}

Under physiological conditions, the expression levels of VEGF and VEGFR in normal human mature tissues very low, and only the expression level of VEGF in vascular endothelial cells is high. However, clinical studies have shown that the high expression of VEGF and VEGFR has been detected in most malignant tumors and is associated with the high risk of cancer metastasis ${ }^{[45]}$. This is because the growth and development of tumors depend on the oxygen and nutrients provided by tumor blood vessels. In highly metabolized tumors, they grow beyond the supply of blood vessels, leading to hypoxia inside the tumor ${ }^{[46]}$. Under hypoxia, hypoxia-inducible factor (HIF-1) regulating angiogenesis is activated, and endothelial cells and tumor cells jointly mediate the upregulation of VEGF expression in $\mathrm{TME}^{[47]}$. VEGF can indirectly promote the inhibition of $\mathrm{T}$ lymphocytes and MDSCs by stimulating endothelial cells to produce prostaglandin E2 (PGE2) $^{[48]}$. Moreover, immunosuppression of tumor microenvironment is caused by inducing the proliferation and differentiation of regulatory $\mathrm{T}$ cells (Tregs) ${ }^{[49]}$ and inhibiting the maturation of dendritic cells (DCs) precursor cells ${ }^{[50]}$.

Apatinib, which is an angiogenesis inhibitor, not only can inhibit Abnormal angiogenesis but also improve the tumor immune microenvironment. According to clinical statistics, at present, there are 33 clinical studies on Apatinib, and among them, there are 29 carried out in China. Comprehensive clinical trial results show that the combination of Apatinib and PD-1/PD-L1 antibody can effectively improve the efficacy of immunotherapy and significantly improve the survival benefit of patients. As a new type of antitumor drug, Apatinib has its superior development prospect and potential. In this paper, we summarized the effects of Apatinib on various immune cells in the tumor microenvironment and summarized the beneficial effects of Apatinib combined with PD-1/PD-L1 antibodies in different kinds of cancer in the clinical trials.

\section{Conflict of interest}

The authors declare no potential conflicts of interest.

\section{References}

1. Hoeksema MA, Gijbels MJ, Velden S, et al. Targeting macrophage Histone deacetylase 3 stabilizes atherosclerotic lesions. EMBO Molecular Medicine 2014; 6(9): 1124-1132.

2. Zhao L, Zhang W, Li C, et al. Research progress on antitumor mechanism of Apatinib. Cancer Research on Prevention and Treatment 2021; 48(1): 7-11.

3. Li Y, Zhao H, Ren X. Relationship of VEGF/VEGFR with immune and cancer cells: Staggering or forward? Cancer Biology \& Medicine 2016; 13(2): 206-214.

4. Ragunathrao VAB, Anwar M, Akhter MZ, et al. Sphingosine-1-phosphate receptor 1 activity promotes tumor growth by amplifying VEGF-VEGFR2 angiogenic signaling. Cell Reports 2019; 29(11): 3472-3487.

5. Maroufin F, Rashidi MR, Vahedian V, et al. Therapeutic potentials of Apatinib in cancer treatment: Possible mechanisms and clinical relevance. Life Sciences 2020; 241: 117106.

6. $\quad$ Mi Y, Liang Y, Huang H, et al. Apatinib (YN968D1) reverses multidrug resistance by inhibiting the efflux function of multiple ATP-binding cassette transporters. Cancer Research 2010; 70(20): 7981-7991.

7. Xu J, Zhang Y, Jia R, et al. Anti-PD-1 antibody SHR-1210 combined with Apatinib for advanced hepatocellular carcinoma, gastric, or esophagogastric junction cancer: An open-label, dose escalation and expansion study. Clinical Cancer Research 2019; 25(2): 515-523.

8. Zheng Y, Yang X, Yan C, et al. Effect of Apatinib plus neoadjuvant chemotherapy followed by resection on pathologic response in patients with locally advanced gastric adenocarcinoma: A single-arm, open-label, phase II trial. European Journal of Cancer 2020; 130: 12-19.

9. Liu C, Jia Q, Wei H, et al. Apatinib in patients with 
advanced chordoma: A single-arm, single-centre, phase 2 study. Lancet Oncology 2020; 21(9): 12441252.

10. Ribas A, Wolchok JD. Cancer immunotherapy using checkpoint blockade. Science 2018; 359(6382): 1350-1355.

11. Lyon AR, Yousaf N, Battisti NML, et al. Immune checkpoint inhibitors and cardiovascular toxicity. Lancet Oncology 2018; 19(9): e447-e458.

12. Wei SC, Aang NAS, Sharma R, et al. Combination anti-CTLA-4 plus anti-PD-1 checkpoint blockade utilizes cellular mechanisms partially distinct from monotherapies. Proceedings of the National Academy of Sciences 2019; 116(45): 22699-22709.

13. Wang DY, Salem JE, Cohen JV, et al. Fatal toxic effects associated with immune checkpoint inhibitors: A systematic review and meta-analysis. JAMA Oncology 2018; 4(12): 1721-1728.

14. Wei F, Wu Y, Tang L, et al. BPIFB1 (LPLUNC1) inhibits migration and invasion of nasopharyngeal carcinoma by interacting with VTN and VIM. British Journal of Cancer 2018; 118(2): 233-247.

15. Vitale I, Manic G, Coussens LM, et al. Macrophages and metabolism in the tumor microenvironment. Cell Metabolism 2019; 30(1): 36-50.

16. Shemesh CS, Hsu JC, Hosseini I, et al. Personalized cancer vaccines: Clinical landscape, challenges and opportunities. Molecular Therapy 2021; 29(2): 555-570.

17. McDermott DF, Huseni MA, Atkins MB, et al. Clinical activity and molecular correlates of response to atezolizumab alone or in combination with bevacizumab versus sunitinib in renal cell carcinoma. Nature Medicine 2018; 24(6): 749-757.

18. Wallin JJ, Bendell JC, Funke R, et al. Atezolizumab in combination with bevacizumab enhances anti-gen-specific T-cell migration in metastatic renal cell Carcinoma. Nature Communications 2016; 7: 12624.

19. Allen E, Jabouille A, Rivera LB, et al. Combined antiangiogenic and anti-PD-L1 therapy stimulates tumor immunity through HEV formation. Science Translational Medicine 2017; 9(385): eaak9679.

20. Tada Y, Togashi Y, Kotani D, et al. Targeting VEGFR2 with Ramucirumab strongly impacts ef- fector/activated regulatory $\mathrm{T}$ cells and $\mathrm{CD} 8^{+} \mathrm{T}$ cells in the tumor microenvironment. Journal for Immunotherapy of Cancer 2018; 6(1): 106.

21. Almeida PED, Mak J, Hernandez G, et al. Anti-VEGF treatment enhances $\mathrm{CD} 8^{+} \mathrm{T}$-cell antitumor activity by amplifying hypoxia. Cancer Immunol Research 2020; 8(6): 806-818.

22. Zhao S, Ren S, Jiang $\mathrm{T}$, et al. Low-dose Apatinib optimizes tumor microenvironment and potentiates antitumor effect of PD-1/PD-L1 blockade in lung cancer. Cancer Immunol Research 2019; 7(4): 630643.

23. Xu Y, Zhang X, Wang Y, et al. A VEGFR2-MICA bispecific antibody activates tumor-infiltrating lymphocytes and exhibits potent anti-tumor efficacy in mice. Cancer Immunology, Immunotherapy 2019; 68(9): 1429-1441.

24. Li Q, Wang Y, Jia W, et al. Low-dose Anti-angio-genic therapy sensitizes breast cancer to PD-1 blockade. Clinical Cancer Research 2020; 26(7): 1712-1724.

25. Huang Y, Yuan J, Righi E, et al. Vascular normalizing doses of antiangiogenic treatment reprogram the immunosuppressive tumor microenvironment and enhance immunotherapy. Proceedings of the National Academy of Sciences of the United States of America 2012; 109(43): 17561-17566.

26. Horikawa N, Abiko K, Matsumura N, et al. Expression of vascular endothelial growth factor in ovarian cancer inhibits tumor immunity through the accumulation of myeloid-derived suppressor cells. Clinical Cancer Research 2017; 23(2): 587-599.

27. Hori S, Nomura T, Sakaguchi S. Control of regulatory $\mathrm{T}$ cell development by the transcription factor Foxp3. Science 2003; 299(5609): 1057-1061.

28. Suzuki H, Onishi H, Wada J, et al. VEGFR2 is selectively expressed by FOXP3high CD4 $4^{+}$Treg. European Journal of Immunology 2010; 40(1): 197203.

29. Terme M, Pernot S, Marcheteau E, et al. VEGFA-VEGFR pathway blockade inhibits tumor-induced regulatory T-cell proliferation in colorectal cancer. Cancer Research 2013; 73(2): 539549.

30. Yasuda S, Sho M, Yamato I, et al. Simultane- 
ous blockade of programmed death 1 and vascular endothelial growth factor receptor 2 (VEGFR2) induces synergistic anti-tumour effect in vivo. Clinical \& Experimental Immunology 2013; 172(3): 500-506.

31. Shigeta K, Datta M, Hato T, et al. Dual programmed death receptor-1 and vascular endothelial growth factor receptor-2 blockade promotes vascular vormalization and enhances antitumor immune responses in hepatocellular carcinoma. Hepatology 2020; 71(4): 1247-1261.

32. Gao C, Jiang Z, Wang G. Mechanism of macrophage polarization in breast cancer associated microenvironment. Chinese Clinical Oncology 2019; 24(3): 274-280.

33. Hato T, Zhu AX, Duda DG. Rationally combining anti-VEGF therapy with checkpoint inhibitors in hepatocellular carcinoma. Immunotherapy 2016; 8(3): 299-313.

34. Alfaro C, Suarez N, Gonzalez A, et al. Influence of bevacizumab, sunitinib and sorafenib as single agents or in combination on the inhibitory effects of VEGF on human dendritic cell differentiation from monocytes. British Journal of Cancer 2009; 100(7): 1111-1119.

35. Dikov MM, Ohm JE, Ray N, et al. Differential roles of vascular endothelial growth factor receptors 1and 2 in dendritic cell differentiation. Journal of Immunology 2005; 174(1): 215-222.

36. Mimura K, Kono K, Takahashi A, et al. Vascular endothelial growth factor inhibits the function of human mature dendritic cells mediated by VEGF receptor-2. Cancer Immunol Immunother 2007; 56(6): 761-770.

37. Cavalheiro ML, Lorena P, Patricia S, et al. Vascular endothelial growth factor-A enhances indoleamine 2, 3-dioxygenase expression by dendritic cells and subsequently impacts lymphocyte proliferation. Memorias Do Instituto Oswaldo Cruz 2014; 109(1): 7079.

38. Ilkovitch D, Lopez DM. The liver is a site for tumor-induced myeloid-derived suppressor cell accumulation and immunosuppression. Cancer Research 2009; 69(13): 5514-5521.

39. Hoechst B, Voigtlaender T, Ormandy L, et al. Mye- loid derived suppressor cells inhibit natural killer cells in patients with hepatocellular carcinoma via the NKp30 receptor. Hepatology 2009; 50(3): 799807.

40. Solito S, Falisi E, Diaz-Montero CM, et al. A human promyelocytic-like population is responsible for the immune suppression mediated by myeloid-derived suppressor cells. Blood 2011; 118(8): 2254-2265.

41. Wu F, Zhang S, Xiong A, et al. A Phase II clinical trial of Apatinib in pretreated advanced non-squamous non-small-cell lung cancer. Clinical Lung Cancer 2018; 19(6): e831-e842.

42. Xu J, Shen J, Gu S, et al. Camrelizumab in combination with Apatinib in patients with advanced hepatocellular carcinoma (RESCUE): A nonrandomized, open-label, phase II trial. Clinical Cancer Research 2021; 27(4): 1003-1011.

43. Li J, Qin S, Xu J, et al. Randomized, double-blind, placebo-controlled phase III trial of Apatinib in patients with chemotherapy-refractory advanced or metastatic adenocarcinoma of the stomach or gastroesophageal junction. Journal of Clinical Oncology 2016; 34(13): 1448-1454.

44. Liu J, Liu Q, Li Y, et al. Efficacy and safety of camrelizumab combined with Apatinib in advanced triple-negative breast cancer: An open-label phase II trial. Journal for Immuno Therapy of Cancer 2020; 8(1): e000696.

45. Takahashi Y, Kitadai Y, Bucana CD, et al. Expression of vascular endothelial growth factor and its receptor, KDR, correlates with vascularity, metastasis, and proliferation of human colon cancer. Cancer Research 1995; 55(18): 3964-3968.

46. Park JS, Kim IK, Han S, et al. Normalization of tumor vessels by Tie2 activation and Ang2 inhibition enhances drug delivery and produces a favorable tumor microenvironment. Cancer Cell 2016; 30(6): 953-967.

47. Kim Y, Nam HJ, Lee J, et al. Methylation-dependent regulation of HIF-1 $\alpha$ stability restricts retinal and tumour angiogenesis. Nature Communications 2016; 7: 10347.

48. Mulligan JK, Rosenzweig SA, Young MRI. Tumor secretion of VEGF induces endothelial cells to sup- 
press $\mathrm{T}$ cell functions through the production of PGE2. Journal of Immunotherapy 2010; 33(2):

126-135.

49. Jayaraman P, Parikh F, Lopez-Rivera E, et al. Tumor-expressed inducible nitric oxide synthase controls induction of functional myeloid-derived suppressor cells through modulation of vascular endothelial growth factor release. Journal of Immunology 2012; 188(11): 5365-5376.

50. Hansen W, Hutzler M, Abel S, et al. Neuropilin 1 deficiency on $\mathrm{CD}^{+}{ }^{+} \mathrm{Foxp}^{+}$regulatory T cells impairs mouse melanoma growth. The Journal of Experimental Medicine 2012; 209(11): 2001-2016. 